\title{
DEGRADAÇÃO DO HERBICIDA AMICARBAZONA ATRAVÉS DE ATIVAÇÃO DE PERSULFATO COM FERRO DE VALÊNCIA ZERO
}

\author{
L. T. N. FUGITA ${ }^{1}$, C. A. L. GRAÇA ${ }^{1}$ e A.C. S. C. TEIXEIRA ${ }^{1}$ \\ ${ }^{1}$ Universidadede São Paulo, Departamento de Engenharia Química \\ E-mail para contato: lucas.fugita@usp.br
}

\begin{abstract}
RESUMO - A presença de quantidades alarmantes de contaminantes emergentes no meio ambiente tem alertado a comunidade científica para a necessidade de desenvolver tecnologias alternativas capazes de remover estes contaminantes, uma vez que os processos convencionais são insuficientes. Neste contexto, os Processos Oxidativos Avançados (POA) têm-se revelado alternativas promissoras. De entre os POA mais aplicados estão os que se baseiam na reação de Fenton $\left(\mathrm{Fe}^{2+}+\mathrm{H}_{2} \mathrm{O}_{2}\right)$, porém estes processos apresentam algumas limitações operacionais, como, por exemplo, a reduzida faixa de $\mathrm{pH}$ em que se pode trabalhar. Dessa maneira, a utilização de um fonte sólida de ferro em vez de Fe(II) aparenta ser uma solução para tal limitação. Posto isto, neste trabalho foi estudada a reação tipo Fenton, baseada na reação entre uma fonte sólida de ferro - o ferro de valência zero (FVZ) - e um oxidante recentemente explorado - o persulfato (PS). $\mathrm{O}$ poluente modelo neste estudo foi o pesticida amicarbazona (AMZ). Avaliou-se o efeito da dosagem inicial de FVZ e PS, bem como do $\mathrm{pH}$, permitindo concluir que o processo $\mathrm{PS} / \mathrm{FVZ}$ é promissor para a remediação de águas contaminadas com AMZ.
\end{abstract}

\section{INTRODUÇÃO}

Processos do tipo Fenton, baseados na reação entre uma fonte de ferro e um oxidante forte (como $\mathrm{H}_{2} \mathrm{O}_{2}$, por exemplo), estão entre os Processos Oxidativos Avançados (POA) mais estudados para tratamento de efluentes (Babuponnusami e Muthukumar, 2014). Este processo provou degradar com sucesso fármacos (Trovó et al. 2009; Trovó et al. 2011), pesticidas (MacKul'Ak et al., 2011), hormônios (Xu et al,. 2009), corantes (Sohrabi et al., 2014) etc. POA convencionais são baseados na produção de radicais hidroxila $\left(\mathrm{HO}^{\circ}\right)$, uma espécie oxidante poderosa que ataca pouco seletivamente matéria orgânica. Recentemente, POA mediados por radicais $\mathrm{SO}_{4}{ }^{--}$têm recebido especial atenção devido às suas vantagens: os reagentes utilizados para geração de $\mathrm{SO}_{4}{ }^{-}$são mais baratos e se dispersam por distâncias maiores (Zhang et al., 2014); $\mathrm{SO}_{4}{ }^{-}$são menos propensos a serem sequestrados por outras moléculas orgânicas (Ji et al., 2015) e após a oxidação são transformados em íons sulfato, ambientalmente amigáveis (Zhang et al., 2014). Um dos reagentes mais utilizados para gerar $\mathrm{SO}_{4}{ }^{-}$é o íon persulfato $\left(\mathrm{S}_{2} \mathrm{O}_{8}{ }^{2-}\right)$, o qual pode ser ativado por calor, bases, radiação UVe metais de transição. $\mathrm{O}$ ferro na forma de íon ferroso $\left(\mathrm{Fe}^{2+}\right)$ tem sido o metal mais utilizado para ativação do PS (Equação 1) devido a sua abundância, baixo custo e propriedades não 
tóxicas (Graça et al., 2017). Sendo assim, este processo pode ser considerado um processo do tipo Fenton.

$$
\mathrm{Fe}^{2+}+\mathrm{S}_{2} \mathrm{O}_{8}{ }^{2-} \rightarrow \mathrm{Fe}^{3+}+\mathrm{SO}_{4}{ }^{2-}+\mathrm{SO}_{4}{ }^{\cdot-}
$$

Contudo, quando o $\mathrm{Fe}^{2+}$ transforma-se em $\mathrm{Fe}^{3+}$ a ativação do PS termina, então grandes quantidades de $\mathrm{Fe}^{2+}$ são necessárias para compensar esse efeito, o que consiste em uma desvantagem desse processo.

Por essas razões, fontes alternativas de $\mathrm{Fe}^{2+}$, tais como magnetita (Avetta et al., 2015) e complexos de Fe(III) (Graça et al., 2017) têm sido exploradas para ativação do PS de maneira mais eficiente por meio da reciclagem de $\mathrm{Fe}^{2+}$ ao longo da reação. $\mathrm{Na}$ ausência de luz, o FVZ pode ser uma alternativa para induzir a ativação do PS, uma vez que a corrosão deste material em meio aquoso pode liberar $\mathrm{Fe}^{2+}$ gradualmente (Equações 2 e 3), permitindo a ativação do PS por períodos mais extensos.

$$
\begin{aligned}
& 2 \mathrm{Fe}^{0}+\mathrm{O}_{2}+2 \mathrm{H}_{2} \mathrm{O} \rightarrow 2 \mathrm{Fe}^{2+}+4 \mathrm{OH}^{-} \\
& \mathrm{Fe}^{0}+2 \mathrm{H}_{2} \mathrm{O} \rightarrow \mathrm{Fe}^{2+}+2 \mathrm{OH}^{-}+\mathrm{H}_{2}
\end{aligned}
$$

Dado isso, neste estudo avaliou-se a degradação do herbicida amicarbazona (AMZ), por meio da ativação do PS com FVZ, explorando-se o efeito das dosagens iniciais de FVZ e PS, bem como do $\mathrm{pH}$.

\section{MATERIAIS E MÉTODOS}

\subsection{Reagentes}

Amostras de grau técnico (> 95,4\% m/m) e analítico $(99,9 \% \mathrm{~m} / \mathrm{m}$ ) de AMZ foram cedidas pela Arysta LifeScience Corp. e utilizadas sem purificação posterior. Todas as soluções aquosas foram preparadas em água Milli-Q.

Micro partículas de FVZ foram adquiridas da J.T. Baker (USA). As partículas possuem área superficial de $3,092 \mathrm{~m}^{2} \mathrm{~g}^{-1}$ (determinada por adsorção isotérmica de $\mathrm{N}_{2}$ ). Todos os demais produtos químicos eram de qualidade analítica.

\subsection{Procedimentos experimentais}

Todos os experimentos foram realizados em duplicata, em um béquer de $250 \mathrm{~mL}$ ao abrigo da luz externa. A solução foi agitada magneticamente a uma temperatura inferior a $20^{\circ} \mathrm{C}$ usando um banho de água gelada para garantir que o persulfato não fosse ativado pelo calor. Primeiramente, a quantidade desejada de FVZ foi adicionada em $100 \mathrm{~mL}$ de solução contendo $41,4 \mu \mathrm{mol} \mathrm{L} \mathrm{L}^{-1}$ de AMZ. Depois, adicionou-se o PS para iniciar a reação de oxidação. Amostras foram retiradas em tempos determinados, imediatamente filtradas por membranas PVDF 0,22 $\mu \mathrm{m}$ para separar as partículas de FVZ e misturadas com $300 \mu \mathrm{L}$ de $\mathrm{MeOH}$ para parar a reação. Quando necessário, o pH foi ajustado com soluções de $\mathrm{NaOH}$ ou $\mathrm{HClO}_{4}$. 


\subsection{Métodos analíticos}

A concentração de ferro dissolvido foi determinada de acordo com o procedimento padrão de o-fenantronila (Mortatti et al., 1982), e o complexo vermelho formado foi determinado espectrofotometricamente em $510 \mathrm{~nm}$.

A concentração de $\mathrm{AMZ}$ durante os experimentos de degradação foi determinada por HPLC-UV (Shimadzu, Series 20A).

\section{RESULTADOS E DISCUSSÃO}

Experimentos controle na ausência de FVZ ou PS foram realizados, variando a concentração de cada reagente, e nenhuma degradação significativa foi observada durante 24h, confirmando que nenhum dos reagentes isoladamente é responsável pela degradação observada durante o tempo de reação (máximo de 180 minutos).

A presença de óxido de Fe(II) na superfície das micro partículas após experimentos com PS+AMZ foi confirmada por difração de raios-X (DRX). Uma das principais vantagens da utilização do FVZ em vez de Fe(II) para ativar o persulfato é a capacidade do primeiro de gradualmente liberar $\mathrm{Fe}$ (II) ao longo do tempo, o que foi observado para as micro partículas.

\subsection{Efeitos da dosagem inicial de FVZ e PS}

$\mathrm{O}$ efeito da dosagem inicial de FVZ foi investigado adicionando diferentes quantidades de micro FVZ na solução de AMZ contendo 41,4 $\mu \mathrm{mol} \mathrm{L}^{-1}\left(10 \mathrm{mg} \mathrm{L}^{-1}\right)$ do poluente, mantendo a concentração inicial de PS fixa em $1 \mathrm{mmol} \mathrm{L}^{-1}$. Observou-se aumento gradual do $\mathrm{pH}$ ao longo do tempo, o que foi considerado dependente da dosagem inicial de FVZ. O pH começou a aumentar mais cedo para dosagens iniciais maiores de FVZ, o que é esperado, uma vez que a quantidade de $\mathrm{OH}^{-}$liberada pela corrosão do ferro é proporcional à concentração inicial de $\mathrm{Fe}^{0}$ de acordo com as Equações 2 e 3 (Deng et al., 2014). Entretanto, ao longo do tempo o aumento de $\mathrm{pH}$ promoveu a precipitação de $\mathrm{Fe}^{2+}$, o que acabou com sua disponibilidade para reagir com PS (Equação 1) e consequentemente diminuiu a degradação da AMZ (Figura 1). Outro fator que pode ter contribuído para menor remoção de AMZ com o aumento da dosagem de FVZ é o sequestro de $\mathrm{SO}_{4}{ }^{-}$pelas quantidades em excesso de $\mathrm{Fe}^{2+}$, de acordo com a Equação 4, reduzindo a eficiência da degradação (Deng et al., 2014; Wei et al., 2016).

$$
\mathrm{Fe}^{2+}+\mathrm{SO}_{4}^{\cdot-} \rightarrow \mathrm{Fe}^{3+}+\mathrm{SO}^{2-}
$$

Resultados similares foram observados por Wei et al. (2016), para os quais altas dosagens de FVZ diminuíram a eficiência da degradação de bentazon por PS/FVZ. 
Figura 1 - Efeito da dosagem inicial de FVZ na degradação de AMZ pelo processo PS/FVZ. $[\mathrm{AMZ}]_{0}=41,4 \mu \mathrm{mol} \mathrm{L}^{-1} ;[\mathrm{PS}]_{0}=1 \mathrm{mmol} \mathrm{L}^{-1} ; 1<[\mathrm{FVZ}]_{0}<20 \mathrm{mmol} \mathrm{L}^{-1} ; \mathrm{pH}$ livre.

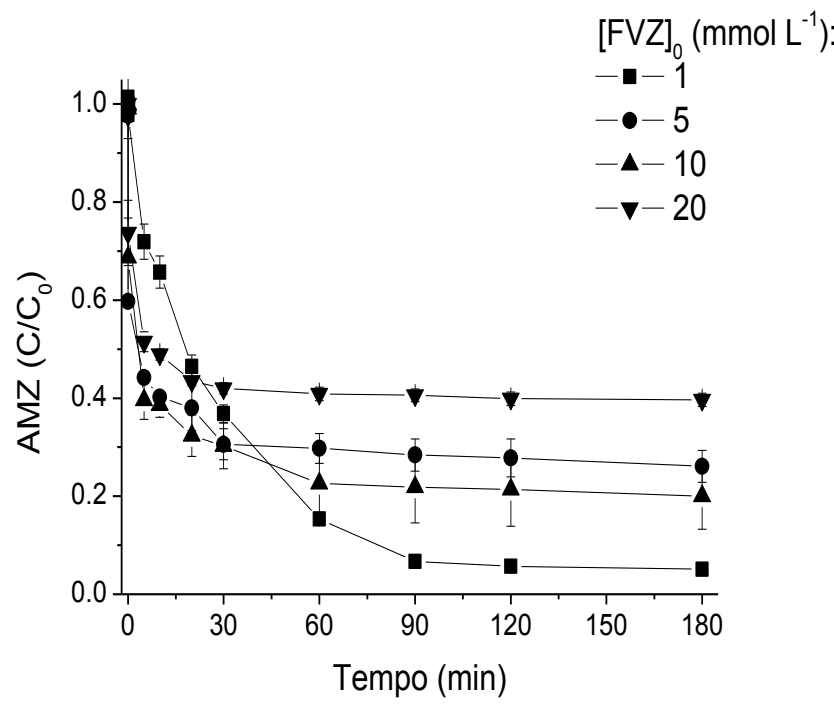

Quanto ao efeito da concentração inicial de PS, a Figura 1 indica que a degradação de AMZ aumenta com o aumento da concentração inicial de PS, o que é esperado uma vez que mais radicais são gerados. Além disso, uma maior concentração inicial de PS causou um aumento mais lento do $\mathrm{pH}$ ao longo do tempo de reação. Como consequência, mais radicais $\mathrm{SO}_{4}{ }^{-}$são produzidos, pois a condição ácida é favorável para a formação e manutenção dos íons ferrosos $\left(\mathrm{Fe}^{2+}\right)$ em solução (Equação 5), portanto existindo mais $\mathrm{Fe}^{2+}$ disponível para reagir com PS.

$$
\mathrm{Fe}^{0}+2 \mathrm{H}^{+} \rightarrow \mathrm{Fe}^{2+}+\mathrm{H}_{2}
$$

Figura 2 - Efeito da concentração inicial de PS na degradação de AMZ pelo processo PS/FVZ. $[\mathrm{AMZ}]_{0}=41,4 \mu \mathrm{mol} \mathrm{L}^{-1} ;[\mathrm{FVZ}]_{0}=5 \mathrm{mmol} \mathrm{L}^{-1} ; 0,5<[\mathrm{PS}]_{0}<2,5 \mathrm{mmol} \mathrm{L}{ }^{-1} ; \mathrm{pH}$ livre.

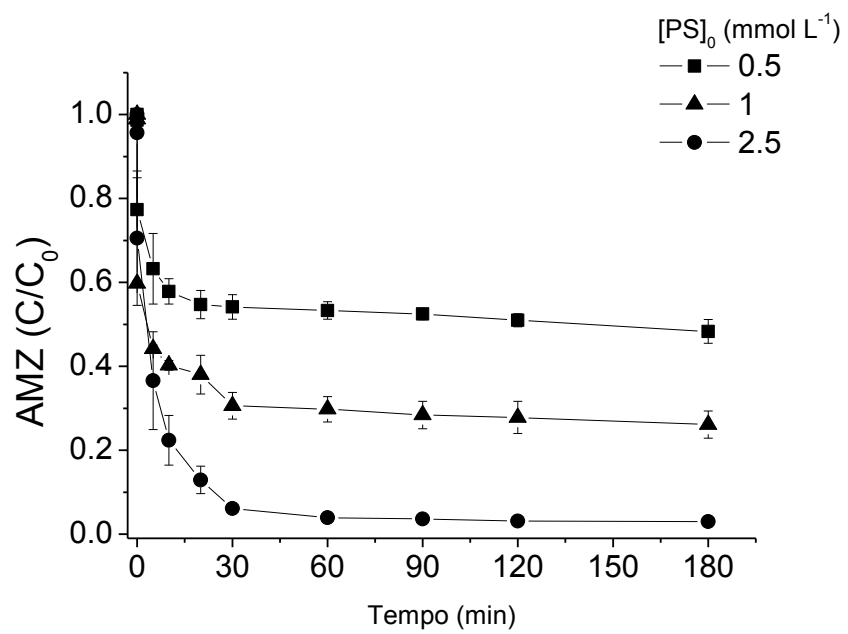


As duas proporções de PS/FVZ que promoveram a maior remoção de AMZ, ou seja, 1:1 e 2,5:5, foram escolhidas para realizar os experimentos descritos a seguir.

\subsection{Efeito do pH da solução}

Para avaliar o efeito do $\mathrm{pH}$ na degradação da AMZ pelo processo PS/FVZ, experimentos foram realizados em três $\mathrm{pH}$ diferentes: 3,$5 ; 5$ e 8 , para as proporções 1:1 e 2,5:5 de PS/FVZ (Figuras 3A e 3B, respectivamente). O sistema PS/FVZ promoveu degradação da $\mathrm{AMZ}$ em ampla variação de $\mathrm{pH}$, embora com menor eficácia em $\mathrm{pH} 8$. Isso acontece devido ao fato de que os íons de ferro serem insolúveis neste $\mathrm{pH}$, formando precipitados e tornando-se indisponíveis para as reações. Mesmo assim, para a maior proporção PS/FVZ, aproximadamente $70 \%$ da AMZ foram removidos em condições alcalinas, indicando que grandes quantidades de FVZ e PS juntos podem superar este inconveniente. Estudos em que $\mathrm{Fe}^{2+}$ é ativado com PS para degradação de poluentes orgânicos mostram que este processo é efetivo apenas em condições ácidas (Rao et al., 2014). Dado isso, os resultados do presente trabalho confirmam que FVZ é muito mais adequado à ativação do PS do que $\mathrm{Fe}^{2+}$, uma vez que permite trabalhar em um intervalo maior de $\mathrm{pH}$. Contudo, íons sulfato presentes em excesso na solução podem sequestrar radicais $\mathrm{HO}^{\circ}$, o que explica o pior desempenho do sistema PS/FVZ em meio alcalino e evidencia a fase de estagnação no perfil de degradação da AMZ para maiores quantidades de PS $\left(2,5 \mathrm{mmol} \mathrm{L}{ }^{-1}\right.$, Figura 3B).

Figura 3 - Efeito do $\mathrm{pH}$ da solução na degradação da AMZ pelo processo PS/FVZ para proporções PS/FVZ de (A) 1:1 e (B) 2,5:0,5 com [AMZ $]_{0}=41,4 \mu \mathrm{mol} \mathrm{L}{ }^{-1}$.
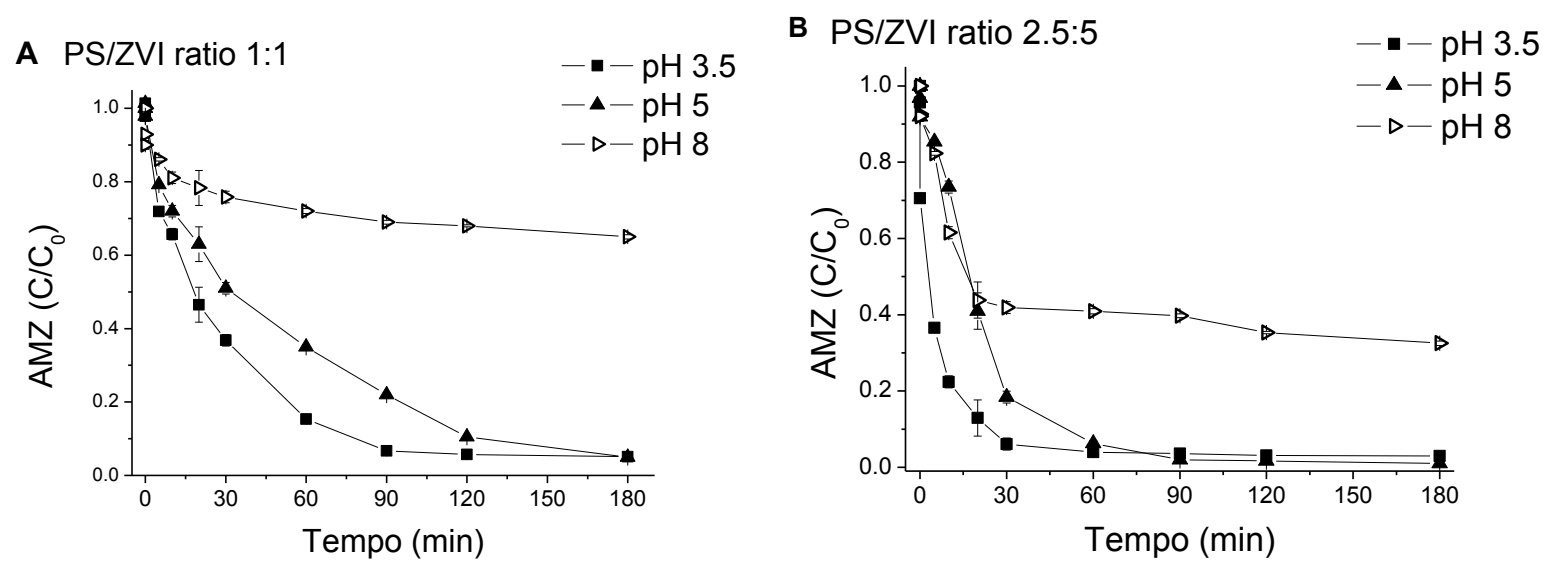

\section{CONCLUSÕES}

Este trabalho permitiu concluir que com o aumento da concentração de FVZ a eficiência do processo PS\FVZ diminui, ao passo que aumentando a concentração de PS, a eficiência aumenta. Variando o pH, verificou-se elevada remoção de AMZ em todo o intervalo estudado ( $\mathrm{pH}$ 3.5-8), embora seja evidente o desempenho pior do processo em condições alcalinas. De um modo geral, o processo aqui estudado permitiu remover eficazmente o poluente alvo, em uma ampla gama de concentrações de reagentes e $\mathrm{pH}$, mostrando-se atrativo para uma possível aplicação para tratamento de águas e efluentes contaminados com AMZ. 


\section{AGRADECIMENTOS}

Os autores agradecem a CAPES-PROEX, CAPES-PROCAD, FAPESP e ao Programa BE-Mundus pelo apoio financeiro. Agradece-se também a Arysta LifeScience Corp, pelo fornecimento das amostras de amicarbazona.

\section{REFERÊNCIAS}

AVETTA P, Implications for the degradation of phenol under heterogeneous photo-fentonlike conditions. Environ Sci Technol, v.49, p.1043-1050, 2015.

BABUPONNUSAMI A,MUTHUKUMAR K. A review on Fenton and improvements to the Fenton process for wastewater treatment. J Environ Chem Eng, v. 2, p. 557-572, 2014.

GRAÇA CAL, VELOSA AC de, TEIXEIRA ACSC. Amicarbazone degradation by UVAactivated persulfate in the presence of hydrogen peroxide or Fe2+. Catal Today, v. 280, p. 80-85, 2017.

JI Y, DONG C, KONG D. Heat-activated persulfate oxidation of atrazine: Implications for remediation of groundwater contaminated by herbicides. Chem Eng J, v. 263, p.45-54, 2015.

MACKUL'AK T, PROSUEK J, ŠVORC L. Degradation of atrazine by Fenton and modified Fenton reactions. Monatshefte fur Chemie v. 142, p. 561-567, 2011.

MORTATTI FJ, KRUG LCR, PESSENDA. Determination of iron in natural waters and plant material with 1,10-phenanthroline by flow injection analysis. Analyst 107, v. 6596, p. 659-663, 1982.

SOHRABI MR, KHAVARAN A, SHARIATI S, SHARIATI S. Removal of Carmoisine edible dye by Fenton and photo Fenton processes using Taguchi orthogonal array design. Arab J Chem., 2014.

TROVÓ AG, NOGUEIRA RFP, AGUERA A. Degradation of sulfamethoxazole in water by solar photo-Fenton. Chemical and toxicological evaluation. Water Res, v. 43, p.39223931, 2009.

TROVÓ AG, PUPO Nogueira RF, AGUERA A. Degradation of the antibiotic amoxicillin by photo-Fenton process - Chemical and toxicological assessment. Water Res, v. 45, p. $1394-$

1402, 2011.

WEI X, GAO N, LI C. Zero-valent iron (ZVI) activation of persulfate (PS) for oxidation of bentazon in water. Chem Eng J, v. 285, p. 660-670, 2016.

XU XR, LI XY, LI XZ, LI H BIN.Degradation of melatonin by $\mathrm{UV}, \mathrm{UV} / \mathrm{H} 2 \mathrm{O} 2, \mathrm{Fe} 2+/ \mathrm{H} 2 \mathrm{O} 2$ and $U V / F e 2+/ H 2 O 2$ processes. Sep Purif Technol, v. 68, p. 261-266, 2009.

ZHANG Q, CHEN J, DAI C. Degradation of carbamazepine and toxicity evaluation using the UV/persulfate process in aqueous solution. J Chem Technol Biotechnol., 2014. 